\title{
Evaluación de las mejores prácticas de manejo (MPM) en el cultivo del palmito (Bactris gasipaes, HBK), en la zona de Santo Domingo
}

Evaluation of the best management practices (MPM) in the cultivation of heart of palm (Bactris gasipaes, HBK), in the Santo Domingo area

Avaliação das melhores práticas de manejo (MPM) no cultivo do palmito (Bactris gasipaes, $H B K)$, na área de Santo Domingo

Freddy Enríquez Jaramillo fgenri5@yahoo.com

Mercedes Charpantier mercds.28@gmail.com

Sofía Segura

segura.sofiac@gmail.com

Camilo Muñoz

camilomunozc12@gmail.com

Juan Ludeña

ludenaj53@gmail.com

Universidad de las Fuerzas Armadas - ESPE Carrera de Ingeniería Agropecuaria Santo Domingo, Ecuador

Artículo recibido septiembre 2018, arbitrado octubre 2018 y publicado en enero 2019

\section{RESUMEN}

El propósito de este artículo es mostrar el estudio de los efectos de las MPM, como una estrategia de manejo sostenible del palmito (Bactris gasipaes HBK), en el que se fomenten niveles óptimos de calidad e inocuidad, protección y respeto por el ambiente. El método empleado para el análisis estadístico fue mediante el diseño de Bloques Completos al Azar (DBCA), en arreglo bifactorial (Tratamientos $\mathrm{X}$ Localidad), con ocho tratamientos (Cuadro 1) y cuatro repeticiones, ubicados en cuatro localidades. Se empleó la prueba de significación de Tukey al $5 \%$ de probabilidad, comparaciones ortogonales entre el Testigo Vs. Resto de tratamientos y Tratamientos fertilizados Vs. Tratamientos no fertilizados. Como resultado se obtuvo que es factible que a mediano plazo al lograr un equilibrio en el desarrollo del cultivo y mejoras en el balance de nutrientes en el suelo se logre una buena producción, ganancias y hasta ahorro que compensa el aumento de los costos de producción.

Palabras clave: Palmito; sostenibilidad ambiente, preservación

\begin{abstract}
The purpose of this article is to show the study of the effects of MPM, as a strategy for the sustainable management of heart of palm (Bactris gasipaes HBK), in which optimum levels of quality and safety, protection and respect for the environment are promoted. The method used for the statistical analysis was through the design of Complete Random Blocks (DBCA), in a bifactorial arrangement (Treatments X Location), with eight treatments (Table 1) and four replications, located in four locations. Tukey's significance test at $5 \%$ probability was used, orthogonal comparisons between the Witness vs. Rest of treatments and Fertilized treatments Vs. Unfertilized treatments. As a result, it was obtained that it is feasible that in the medium term, by achieving a balance in the development of the crop and improvements in the balance of nutrients in the soil, good production, profits and even savings are achieved that compensates for the increase in production costs.
\end{abstract}

Key words: Palmetto; environmental sustainability, preservation

Alfa, Revista de Investigación en Ciencias Agronómicas y Veterinarias Vol. 3, N Nro. 7, Enero - Abril 2019 


\section{RESUMO}

O objetivo deste artigo é mostrar o estudo dos efeitos do MPM, como estratégia para o manejo sustentável do palmito (Bactris gasipaes HBK), no qual são promovidos níveis ótimos de qualidade e segurança, proteção e respeito ao meio ambiente. 0 método utilizado para a análise estatística foi o delineamento de Blocos Aleatórios Completos (DBCA), em arranjo bifatorial (Tratamentos X Localização), com oito tratamentos (Tabela 1) e quatro repetições, localizados em quatro locais. Utilizou-se o teste de significância de Tukey a 5\% de probabilidade, comparações ortogonais entre o Testemunha vs. Resto dos tratamentos e Tratamentos fertilizados vs. Tratamentos não fertilizados. Como resultado, foi possível viabilizar que, a médio prazo, alcançando um equilíbrio no desenvolvimento da safra e melhorias no balanço de nutrientes no solo, sejam alcançadas boa produção, lucros e até economias que compensem o aumento dos custos de produção.

Palavras-chave: Palmito; sustentabilidade ambiental, preservação

\section{INTRODUCCIÓN}

En Ecuador el cultivo de palmito ha experimentado un importante crecimiento dentro de los productos agrícolas no tradicionales, siendo uno de los principales exportadores de palmito a nivel mundial. Debido a esto el productor ha implementado una serie de prácticas agrícolas que no siempre han repercutido en mejorar la productividad del cultivo, en muchos casos se hacen mal o a destiempo, empleando ineficientemente insumos agrícolas, principalmente plaguicidas y fertilizantes.

La intensificación de cualquier cultivo debe responder a los objetivos de rentabilidad, sustentabilidad del sistema de producción y protección del ambiente. Por otra parte, estos deben responder a los objetivos de sustentabilidad económica, ecológica y social comunes a toda la sociedad (Bruuselma, et al., 2008).

Este marco general acentúa la necesidad de aplicar las Mejores Prácticas de Manejo (MPM) empleando principios técnicos que sean probados en el lugar de establecimiento del cultivo, evaluados a través de indicadores que reflejen el efecto combinado de los mismos a nivel local y regional.

La finalidad de la investigación es estudiar los efectos de las MPM, como una estrategia de manejo sostenible del palmito (Bactris gasipaes HBK), en el que se fomenten niveles óptimos de calidad e inocuidad, protección y respeto por el ambiente. Además de evaluar la acción de las MPM sobre ciertas variables agronómicas y rentabilidad de diferentes tratamientos.

\section{MATERIALES Y MÉTODOS}

El proyecto se ejecutó en cuatro localidades: Hcda. Zoila Luz (Sector Luz de América); Hcda. El Chontal (Sector Valle Hermoso Bajo), Hcda. San Jorge (Sector El Recreo- Valle Hermoso Alto); Hcda. La Pisona (Sector La Unión), las tres primeras están ubicadas en el Cantón Santo Domingo y la última en el Cantón Quinindé. 
Cuadro 1. Tratamientos a evaluar en ensayo sobre MPM

\begin{tabular}{cl}
\hline $\begin{array}{c}\text { En palmito } \\
\text { Tratamiento }\end{array}$ & \multicolumn{1}{c}{ Descripción } \\
\hline T0 & Testigo (manejo convencional, la manera en que maneja el agricultor en esta zona.) \\
T1 & Control manual de malezas (machete) \\
T2 & Control manual de malezas (machete) + limpieza de cepa \\
T3 & Control manual de malezas (machete) + limpieza de cepa + deshije \\
T4 & Control manual de malezas (machete) + limpieza de cepa + deshije + deshoje \\
T5 & Control manual de malezas (machete) + limpieza de cepa + deshije + deshoje+ \\
& control de plagas \\
T6 & Control manual de malezas (machete) + limpieza de cepa + deshije + deshoje+ \\
& control de plagas + fertilización \\
T7 & Control manual de malezas (machete) + limpieza de cepa + deshije + deshoje+ \\
& control de plagas \\
& fertilización + cosecha (parámetros de cosecha) \\
\hline
\end{tabular}

Para el análisis estadístico se empleó un Diseño de Bloques Completos al Azar (DBCA), en arreglo bifactorial (Tratamientos $\mathrm{X}$ Localidad), con ocho tratamientos (Cuadro 1) y cuatro repeticiones, ubicados en cuatro localidades. Se empleó la prueba de significación de Tukey al $5 \%$ de probabilidad, comparaciones ortogonales entre el Testigo Vs. Resto de tratamientos y Tratamientos fertilizados Vs. Tratamientos no fertilizados, a más de correlaciones entre variables dependientes e independientes. Las variables evaluadas en cada tratamiento fueron: altura de hijuelos, numero de hijuelos, número de tallos cosechados, concentración de nutrientes a nivel de suelo $y$ foliar, concentración de esporas de micorrizas, análisis Beneficio/Costo.

\section{RESULTADOS Y DISCUSIÓN}

\section{Altura de hijuelos}

Los ADEVAS efectuadas para esta variable muestran diferencias estadísticas para tratamientos y la comparación Tratamientos fertilizados Vs. Tratamientos no fertilizados en los meses de diciembre 2012 y febrero 2013, mientras que para localidades las diferencias estadísticas fueron altamente significativas para todos los meses de evaluación (Tabla 1).

Tabla 1. Cuadrados medios de los análisis de variancia para la altura de hijuelos en diferentes meses mostrando el efecto de la aplicación de las MPM sobre el cultivo de palmito.

\begin{tabular}{lllllll}
\multicolumn{1}{c}{ F.V. } & \multicolumn{6}{c}{ MESES DE EVALUACIÓN } \\
& G.L. & \multicolumn{1}{c}{ Agosto } & \multicolumn{1}{c}{ Octubre } & Noviembre & Diciembre & Febrero \\
\hline REPETICIONES & 3 & $0,07 \mathrm{~ns}$ & $10,09 \mathrm{~ns}$ & $14,94 \mathrm{~ns}$ & $13,88 \mathrm{~ns}$ & $20,23 \mathrm{~ns}$ \\
TRATAMIENTOS & 7 & $4,39 \mathrm{~ns}$ & $5,10 \mathrm{~ns}$ & $27,56 \mathrm{~ns}$ & $50,47^{*}$ & $80,54^{* *}$ \\
LOCALIDAD & 3 & $330,93^{* *}$ & $811,53^{* *}$ & $1358,01^{* *}$ & $510,99^{* *}$ & $251,21^{* *}$ \\
TRATAMIENTOS*LOCALIDAD & 21 & $3,07 \mathrm{~ns}$ & $4,37 \mathrm{~ns}$ & $19,58 \mathrm{~ns}$ & $20,38 \mathrm{~ns}$ & $23,12 \mathrm{~ns}$ \\
Testigo Vs. Resto Trat. & 1 & $1,74 \mathrm{~ns}$ & $3,25 \mathrm{~ns}$ & $10,37 \mathrm{~ns}$ & $29,54 \mathrm{~ns}$ & $55,05 \mathrm{~ns}$ \\
Trat. Fert. Vs. Trat. No fert. & 1 & $1,06 \mathrm{~ns}$ & $10,66 \mathrm{~ns}$ & $56,58 \mathrm{~ns}$ & $165,51^{*}$ & $338,44^{* *}$ \\
Error & 93 & 4,57 & 6,49 & 18,54 & 19,09 & 18,35 \\
Total & 127 & & & & & \\
C.V. (\%) & & 17,67 & 15,34 & 19,30 & 16,90 & 14,70 \\
\hline
\end{tabular}

ns $=$ no significativo, ${ }^{*}=$ significativo, ${ }^{* *}=$ altamente significativo 
Al observar la figura 1 se evidencia que la mayoría de tratamientos donde se aplicaron las MPM ocupan en ambos meses los primeros rangos de significación estadística, sobresaliendo con los mejores promedios el tratamiento T7 (Control manual de malezas (machete) + limpieza de cepa + deshije + deshoje + control de plagas + fertilización + cosecha (parámetros de cosecha)) con valores de 29,60 y 34,11 cm en diciembre y febrero, los promedios más bajos fueron para T0 y $\mathrm{T} 4$. Al parecer la fertilización balanceada no incide mayormente en la altura de hijuelos, pues el tratamiento T6 muestra valores similares a otros donde no se incorporó la fertilización, resultados similares lo obtuvieron Villaprado (2009) y Solano (2012), quienes no encontraron diferencias estadísticas en altura de plantas de palmito fertilizadas con diferentes dosis de N, P y K.

El ensayo ubicado en la Hcda. San Jorge (Valle Hermoso Alto), presentó mejor respuesta en altura de hijuelos, seguida de la localidad La Pisona (Quinindé) a partir de noviembre, Zoila luz presentó en todas las evaluaciones los promedios más bajos (Figura 2).

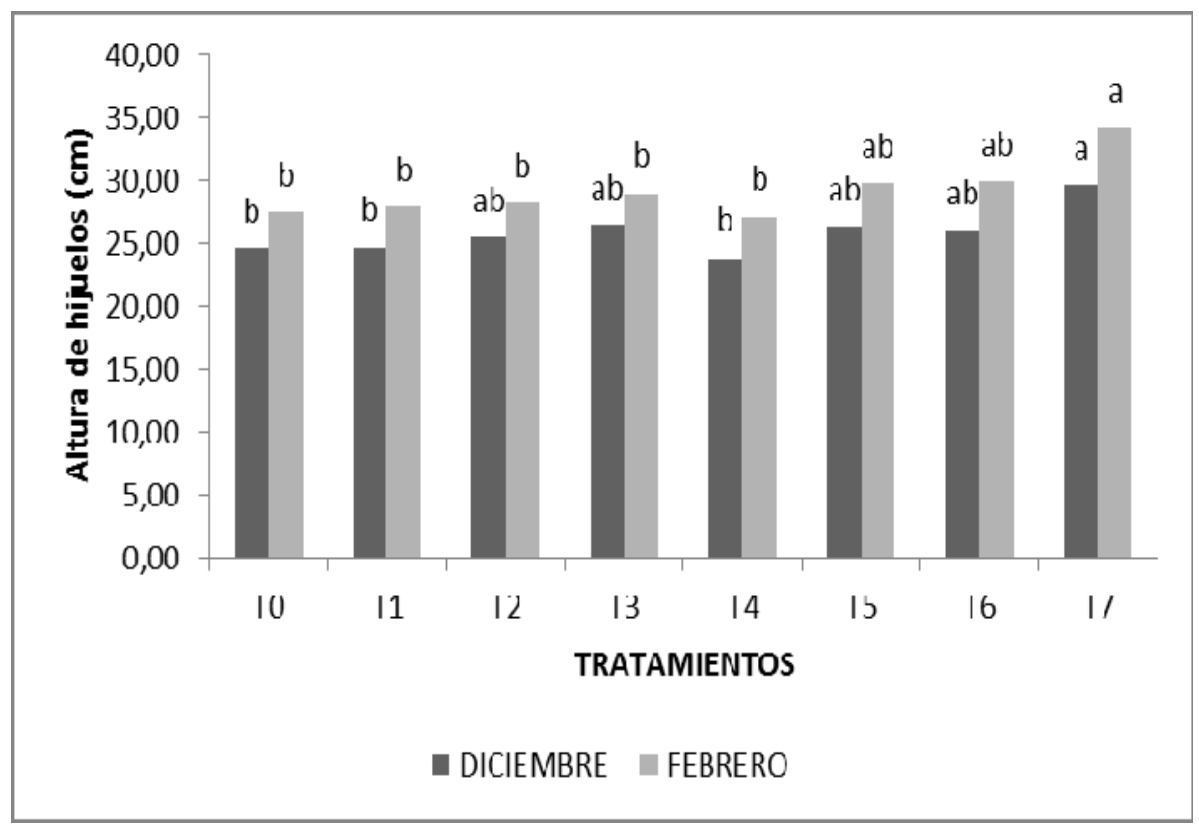

Figura 1. Efecto de la aplicación de las MPM sobre la altura de hijuelos (cm) en el cultivo de palmito en dos épocas de evaluación. 


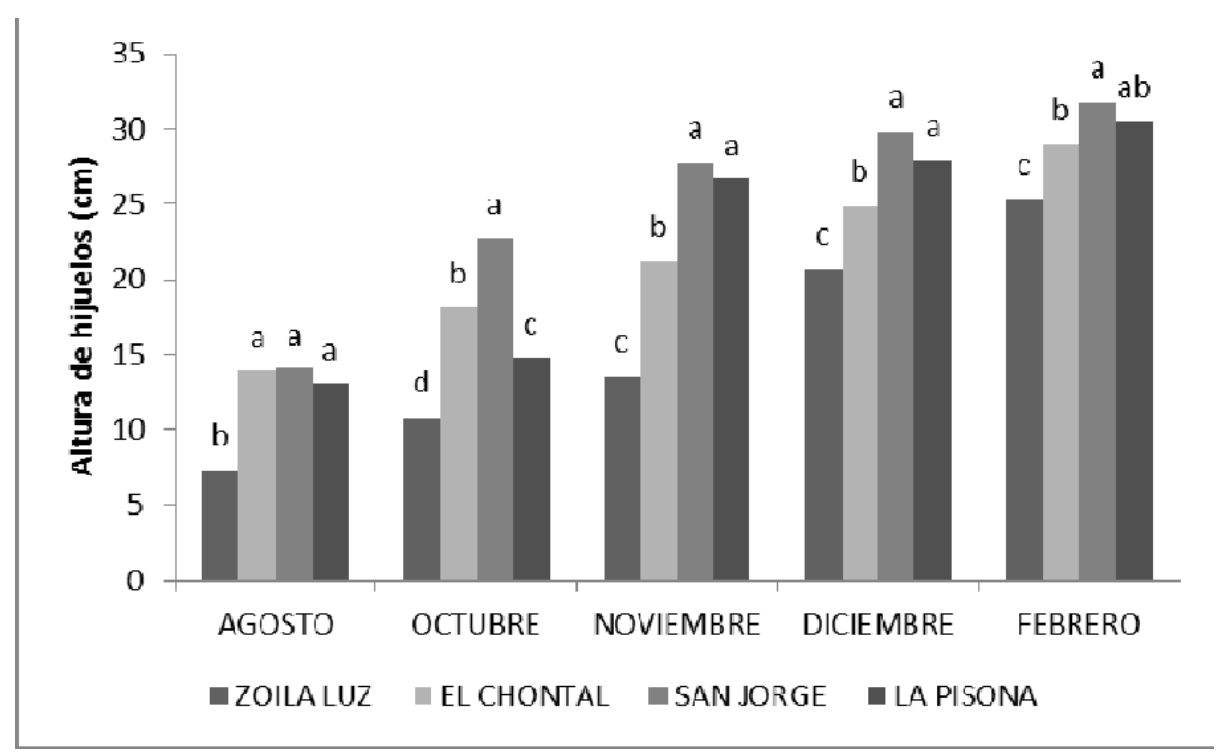

Figura 2. Efecto de la aplicación de las MPM sobre la altura de hijuelos (cm) en el cultivo de palmito en diferentes localidades.

Al comparar los promedios de la figura 3 , se nota que los tratamientos fertilizados superaron a los no fertilizados, tanto en diciembre como en febrero, con valores de 27,82 y $31,97 \mathrm{~cm}$ respectivamente, en relación a 25,20 y 28,21 alcanzados por los tratamientos no fertilizados. Los tratamientos fertilizados incluyeron a T7 y T6, este último a más de la fertilización incorporó parámetros de cosecha que sin duda influyó en el promedio de ambos tratamientos, fisiológicamente la cosecha intensiva de los tallos (rondas semanales) estimulan el crecimiento de yemas y brotes provocando un mejor desarrollo de los hijuelos y por tanto acortando su periodo de cosecha.

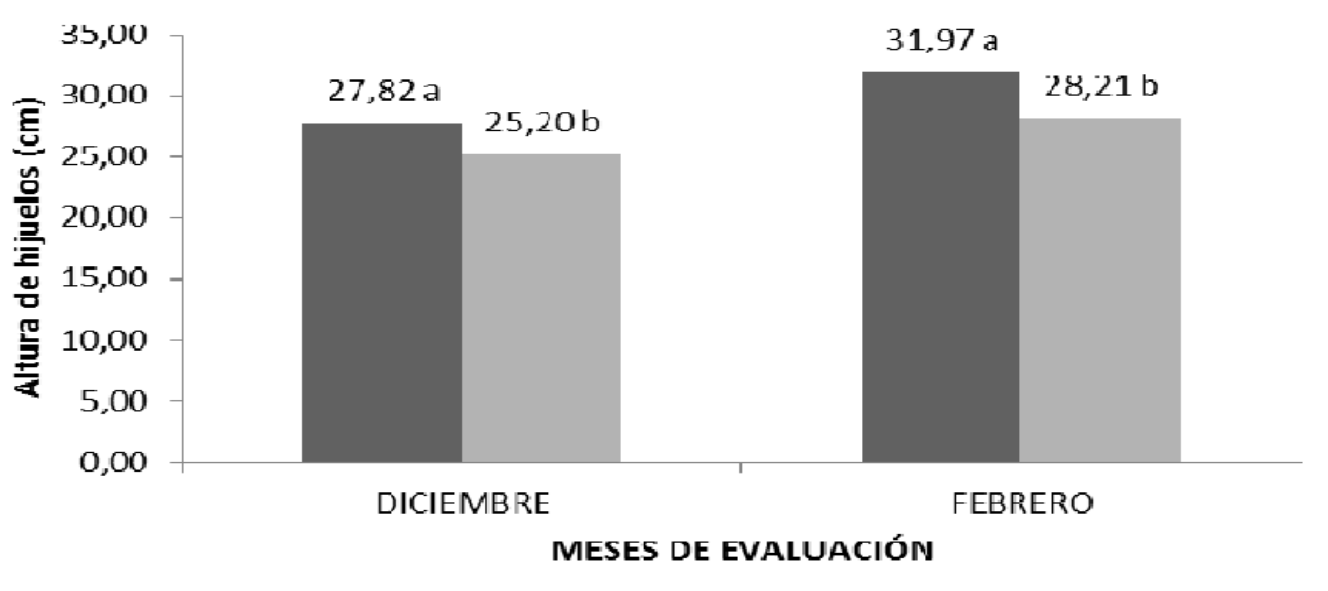

- TRATAMIENTOS FERTILIZADOS TRATAMIENTOS NO FERTILIZADOS

Figura 3. Efecto de la fertilización balanceada sobre la altura de hijuelos (cm) en el cultivo de palmito en dos épocas de evaluación. 


\section{Número de hijuelos}

El ADEVA realizado para el número de hijuelos evaluados al final del ensayo, refleja que hubo diferencias estadísticas para todas las fuentes de variación, tal como se observa en la Tabla 2.

En la figura 4, se determina que los tratamientos estadísticamente iguales corresponden a T5, T6 y T7 con valores de 3,$55 ; 3,54$ y 3,53 hijuelos respectivamente, el primero incluyó el control manual de malezas (machete) + limpieza de cepa + deshije + deshoje+ control de plagas, al segundo tratamiento se le adicionó la fertilización balanceada y al T7 a más de todos ellos se adicionó parámetros de cosecha, el promedio más bajo le correspondió a T2 (Control manual de malezas (machete) + limpieza de cepa) con 2,72 hijuelos aunque estadísticamente es similar a T0, T1 y T3. Lo que implica que a partir del T4 donde ya se adicionó el deshoje se empieza a notar los efectos de las MPM sobre el número de hijuelos, esto concuerda con Mora (1999), quien manifiesta que la poda estimula la formación de hijos en la parte basal del tallo.

Tabla 2. Cuadrado medio del análisis de varianza para la variable número ce Hijuelos, mostrando el efecto de la MPM sobre el cultivo de palmito.

\begin{tabular}{lll}
\hline F.V. & G.L. & CM \\
\hline Repeticiones & 3 & 0,13 \\
Tratamientos & 7 & $1,94^{* *}$ \\
Localidad & 3 & $32,23^{* *}$ \\
Tratamiento * localidad & 21 & $0,61^{*}$ \\
Testigo vs. Resto tratamiento & 1 & $2.14^{*}$ \\
Trat. Fert. Vs. Trat. No fert & 1 & $4,84^{* *}$ \\
Error & 93 & 0,31 \\
Total & 127 & \\
C.V. $(\%)$ & & 17,45 \\
\hline
\end{tabular}

ns= no significativo, ${ }^{*}=$ significativo, ${ }^{* *}=$ altamente significativo

La localidad con mayor número de hijuelos fue Zoila Luz (Luz de América) con un promedio de 4,63 hijuelos, mientras que El Chontal (Valle Hermoso Bajo) tuvo el número más bajo con 2,27 hijuelos.

Para la interacción Tratamiento $\mathrm{X}$ Localidad, se determinó que los tratamientos T5 y T6 ubicados en Zoila Luz presenten el mayor número de hijuelos de 5,25 en ambos casos, el resto de tratamientos si bien difieren en los promedios la mayoría ocupan los mismos rangos de significación estadística.

Los tratamientos donde se aplicaron las MPM (T1-T7) con un promedio de 3,25 hijuelos, superaron al testigo (T0) que presentó un valor de 2,86 hijuelos, asimismo los tratamientos fertilizados (T6 y T7) superaron estadísticamente a los no fertilizados, con promedios de 3,54 y 3,09 hijuelos respectivamente. 


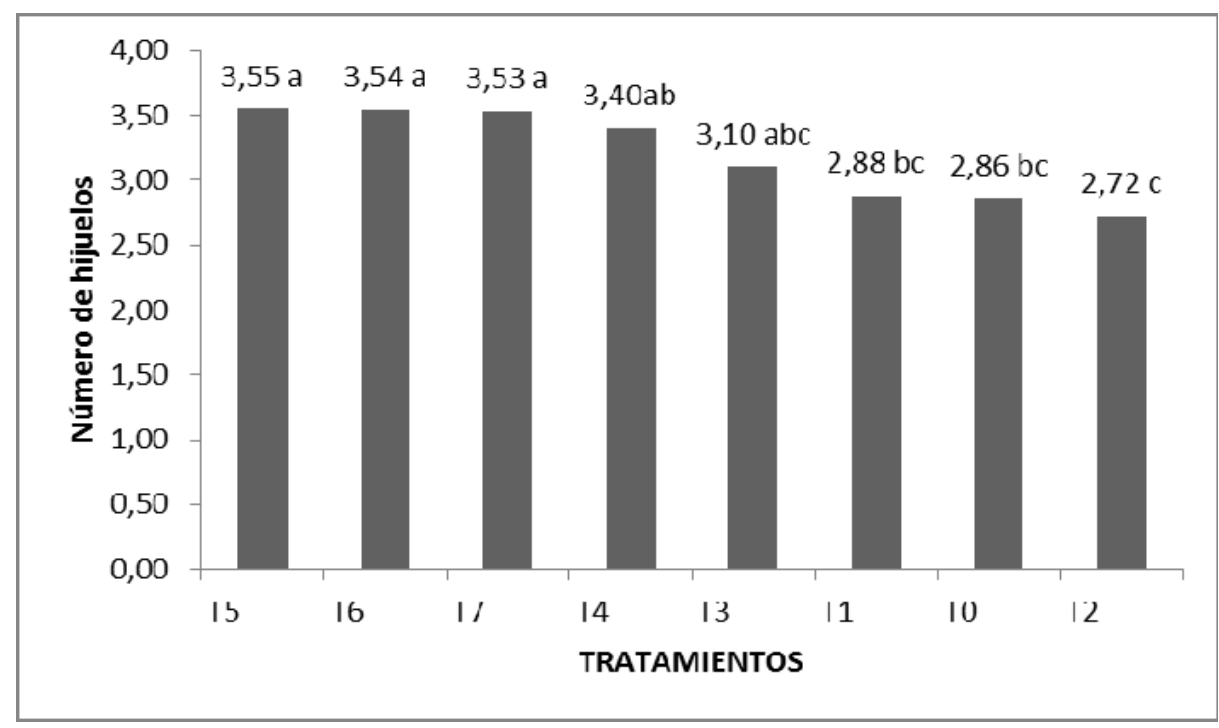

Figura 4. Efecto de la aplicación de las MPM sobre el número de hijuelos en el cultivo de palmito.

2012- 2013.

\section{Número de tallos cosechados}

El resumen del ADEVA de la tabla 3, muestra diferencias estadísticas para todas las fuentes de variación.

El tratamiento que sobresalió estadísticamente fue el T7 con un promedio de 46,31 tallos, en contraste, el valor más bajo fue para T0 con 22,50 tallos cosechados (Figura 6). El tratamiento T3 donde se incorporó el deshije, tuvo uno de los promedios más bajos, contrario a la investigación realizada por Reyes et.al (2003), quienes afirman que, realizando un deshije adecuado y dejando de 4 a 6 hijuelos viables por cepa, se obtiene mayor producción de palmito por hectárea.

Tabla 3. Cuadrado medio del análisis de variancia para la variable número tallos cosechados, mostrando el efecto de las MPM sobre el cultivo de palmito.

\begin{tabular}{lcl}
\hline F.V. & G.L. & \multicolumn{1}{c}{ CM } \\
\hline REPETICIONES & 3 & 25,72 \\
TRATAMIENTOS & 7 & $908,35^{* *}$ \\
LOCALIDAD & 3 & $6676,45^{* *}$ \\
TRATAMIENTOS*LOCALIDAD & 21 & $49,49 *$ \\
Testigo Vs. Resto Trat. & 1 & $744,97^{* *}$ \\
Trat. Fert. Vs. Trat. No fert. & 1 & $4233,40^{* *}$ \\
Error & 93 & 26,64 \\
Total & 127 & \\
C.V. (\%) & \multicolumn{2}{c}{17,45} \\
\hline
\end{tabular}

ns $=$ no significativo,${ }^{*}=$ significativo,${ }^{* *}=$ altamente significativo 
Las localidades con mayores rendimientos fueron El Chontal y Zoila Luz con 42,34 y 40,38 tallos, mientras que los promedios más bajos fueron para La Pisona y San Jorge con 17,22 y 15,59 tallos cosechados.

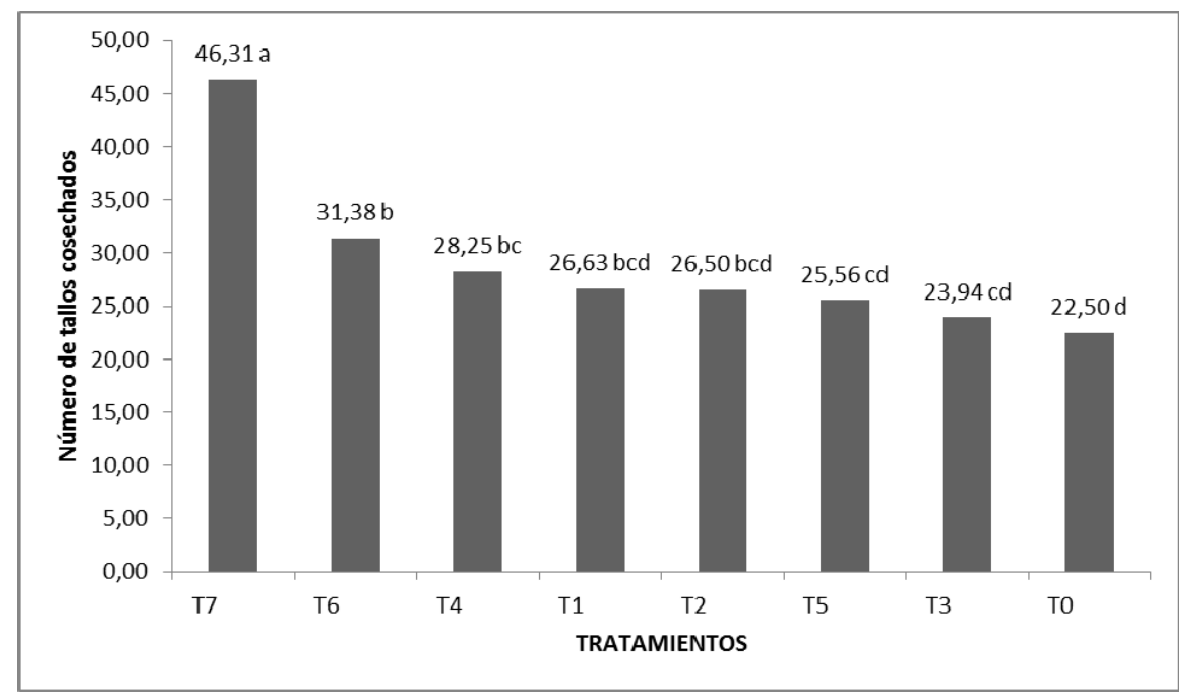

Figura 6. Efecto de la aplicación de las MPM sobre el número de tallos cosechados en el cultivo de palmito.

La interacción Tratamiento X Localidad destacó al tratamiento T7 con los mejores promedios de 57,75 y 53,25 tallos cosechados ubicados en las localidades de Zoila Luz y El Chontal respectivamente, los promedios más bajos los ocuparon algunos tratamientos en diferentes localidades; sin embargo, T0 en la localidad de San Jorge fue el peor promedio con 9,50 tallos cosechados.

Al comparar los tratamientos donde se aplicaron las MPM (T1-T7) con el testigo (T0), se determinó que los primeros superaron a los segundos con valores de 29,80 y 22,50 tallos cosechados respectivamente; del mismo modo, los tratamientos donde a más de otras labores se incorporó la fertilización (T6 y T7), sobresalieron ampliamente sobre los demás, alcanzando un promedio de 38,85 tallos, mientras que el resto tuvo un valor de 25,56 tallos cosechados, lo que coincide con Molina (2000), quienes realizaron una investigación con dosis de nutrientes en plantaciones de palmito de cuatro años de edad, concluyendo que fertilizando el palmito se incrementó significativamente el número de tallos cosechados por ha/año. Resultado similar encontró Villaprado 2009, quien además de manejar técnicamente el cultivo (control de malezas, deshoje, deshije, cosecha oportuna), evaluó la dosis de nutrientes necesarios para el cultivo, afirmando que a medida de que se aumenta la dosis de fertilizantes al cultivo, el número de tallos cosechados se incrementó.

\section{Concentración de nutrientes en el suelo}

Los ADEVAS para esta variable sólo mostraron significación estadística para localidades (Tabla 4). En general los resultados reflejan una mayor disponibilidad de $\mathrm{Ca}, \mathrm{Cu}, \mathrm{B}$ y $\mathrm{Fe}$ en la localidad de Zoila Luz, mientras que el K, P, Zn tuvieron mayor concentración en El Chontal, presumiblemente ésta mejor 
condición de suelo de estas localidades podría haber influido en un mayor número de tallos cosechados, sin descartar que este resultado también evidencie una mejor condición de planta y manejo del cultivo anterior a la implementación de las MPM.

Tabla 4. Cuadrados medios de los análisis de variancia para la concentración de nutrientes en el suelo, mostrando el efecto de la aplicación de las MPM sobre el cultivo de palmito.

\begin{tabular}{|c|c|c|c|c|c|c|c|c|c|}
\hline \multirow[b]{2}{*}{ F.V. } & \multirow[b]{2}{*}{ G.L. } & \multicolumn{8}{|c|}{ NUTRIENTES } \\
\hline & & $\mathbf{P}$ & $\mathbf{K}$ & Ca & $\mathbf{S}$ & $\mathbf{F e}$ & B & $\mathrm{Cu}$ & $\mathbf{Z n}$ \\
\hline LOCALIDAD & 3 & 1,69 & $0,02 * *$ & $6,28 * *$ & $0,08 * *$ & $\begin{array}{c}34265,71 \\
* *\end{array}$ & $0,23^{* *}$ & $\begin{array}{c}42,17 \\
* *\end{array}$ & $0,35^{* *}$ \\
\hline TRATAMIENTOS & 7 & $\begin{array}{c}0,05 \\
\text { ns }\end{array}$ & $\begin{array}{c}0,0018 \\
\text { ns }\end{array}$ & $0,53 \mathrm{~ns}$ & $0,02 \mathrm{~ns}$ & $\begin{array}{c}1222,55 \\
n s\end{array}$ & $0,02 \mathrm{~ns}$ & $\begin{array}{c}1,01 \\
\text { ns }\end{array}$ & $0,03 \mathrm{~ns}$ \\
\hline Testigo Vs. & 1 & 1,10 & 0,0019 & 0,0045 & 0,000025 & 270,10 & 0,00099 & 5,72 & 0,0015 \\
\hline Resto Trat. & & ns & ns & ns & ns & ns & ns & ns & ns \\
\hline Trat. Fert. Vs. & 1 & 0,13 & 0,01 & $0,51 \mathrm{~ns}$ & $0,05 \mathrm{~ns}$ & 1488,30 & $0,04 \mathrm{~ns}$ & 0,84 & 0,00023 \\
\hline $\begin{array}{l}\text { Trat. No fert. } \\
\text { Error }\end{array}$ & 93 & $\begin{array}{c}\text { ns } \\
0,03\end{array}$ & $\begin{array}{c}\mathrm{ns} \\
3,20 \mathrm{E}- \\
03\end{array}$ & $1,19 \mathrm{E}+00$ & 0,01 & $\begin{array}{c}\text { ns } \\
1120,9\end{array}$ & 0,02 & $\begin{array}{c}\text { ns } \\
1,51\end{array}$ & $\begin{array}{c}\mathrm{ns} \\
0,02\end{array}$ \\
\hline $\begin{array}{c}\text { Total } \\
\text { C.V. (\%) }\end{array}$ & 127 & 15,16 & 20,89 & 17,51 & 9,02 & 10,96 & 19,31 & 15,61 & 19,68 \\
\hline
\end{tabular}

$n s=$ no significativo, ${ }^{*}=$ significativo,${ }^{* *}=$ altamente significativo

\section{Concentración foliar de nutrientes}

El resumen de los ADEVAS (Tabla 5) para esta variable, determinaron diferencias estadísticas altamente significativas únicamente para localidades en todos los nutrientes, demostrando las diferencias en cuanto ha estado nutritivo de las plantas que es un reflejo de la fertilidad del suelo en cada localidad estudiada.

Al analizar los promedios en cada localidad, se determina que el sitio San Jorge (Valle Hermoso Alto) presentó mayor concentración foliar de nutrientes particularmente $\mathrm{K}, \mathrm{Ca}, \mathrm{P}, \mathrm{S}, \mathrm{B}, \mathrm{Fe}$ y $\mathrm{Mn}$, mientras que la localidad Zoila Luz tuvo la concentración más baja de $\mathrm{K}, \mathrm{P}, \mathrm{Mg}, \mathrm{S}, \mathrm{B}, \mathrm{Fe}$ y $\mathrm{Mn}$, aparentando un contrasentido ya que ésta última fue una de las de mejor comportamiento agronómico, específicamente en número de hijuelos y tallos cosechados. Esto se entendería por la dilución de contenido foliar (efecto Steenjberg) debido al mayor crecimiento exhibido por las plantas de Zoila Luz (Malavolta, 1994; Silva, 2001; Correndo y García, 2012). 
Tabla 5. Cuadrados medios de los análisis de variancia para la concentración foliar de nutrientes, mostrando el efecto de la aplicación de las MPM, sobre el cultivo de palmito.

\begin{tabular}{|c|c|c|c|c|c|c|c|c|c|c|c|c|}
\hline F.V. & & & & & & NUT & IENTES & & & & & \\
\hline G.L. & & $\mathbf{N}$ & $\mathbf{P}$ & $\mathbf{K}$ & $\mathrm{Ca}$ & Mg & $\mathbf{S}$ & $\mathbf{C u}$ & B & $\mathbf{F e}$ & Zn & Mn \\
\hline REPETICIONES & 3 & $\begin{array}{l}0,11 \\
\text { ns }\end{array}$ & $\begin{array}{l}0,01 \\
\text { ns }\end{array}$ & 0,21 ** & $0,03 *$ & $0,04 *$ & $0,02 \mathrm{~ns}$ & $0,0032 \mathrm{~ns}$ & $0,13^{* *}$ & $0,14^{* *}$ & $0,01 \mathrm{~ns}$ & $0,08^{* *}$ \\
\hline TRATAMIENTOS & 7 & $\begin{array}{l}0,16 \\
\mathrm{~ns}\end{array}$ & $\begin{array}{l}0,01 \\
\mathrm{~ns}\end{array}$ & $0,02 \mathrm{~ns}$ & $0,003 \mathrm{~ns}$ & $0,004 \mathrm{~ns}$ & $0,01 \mathrm{~ns}$ & $0,04 \mathrm{~ns}$ & $0,01 \mathrm{~ns}$ & $0,01 \mathrm{~ns}$ & $0,0033 \mathrm{~ns}$ & $0,01 \mathrm{~ns}$ \\
\hline LOCALIDAD & 3 & $\begin{array}{l}1,42 \\
* *\end{array}$ & $\begin{array}{l}0,13 \\
* *\end{array}$ & $6,41^{* *}$ & $0,21^{* *}$ & 0,92 ** & 0,22 ** & $0,04 \mathrm{~ns}$ & $0,25^{* *}$ & $0,83^{* *}$ & $0,11^{* *}$ & $0,87^{* *}$ \\
\hline $\begin{array}{l}\text { TRATAMIENTOS* } \\
\text { LOCALIDAD }\end{array}$ & 21 & $\begin{array}{l}0,28 \\
\mathrm{~ns}\end{array}$ & $\begin{array}{l}0,01 \\
\text { ns }\end{array}$ & $0,01 \mathrm{~ns}$ & $0,005 \mathrm{~ns}$ & $0,0031 \mathrm{~ns}$ & $0,01 \mathrm{~ns}$ & 0,05 * & $0,03 \mathrm{~ns}$ & $0,02 \mathrm{~ns}$ & $0,01 \mathrm{~ns}$ & $0,01 \mathrm{~ns}$ \\
\hline Testigo Vs. Resto Trat. & 1 & $\begin{array}{l}0,20 \\
\mathrm{~ns}\end{array}$ & $\begin{array}{l}0,01 \\
\text { ns }\end{array}$ & $0,04 \mathrm{~ns}$ & $0,003 \mathrm{~ns}$ & $0,0022 \mathrm{~ns}$ & $0,0033 \mathrm{~ns}$ & $0,02 \mathrm{~ns}$ & $0,00001 \mathrm{~ns}$ & $0,0016 \mathrm{~ns}$ & $0,000048 \mathrm{~ns}$ & $0,0035 \mathrm{~ns}$ \\
\hline Trat. Fert. Vs. Trat. No fert. & 1 & $\begin{array}{l}0,07 \\
\text { ns }\end{array}$ & $\begin{array}{l}0,01 \\
\text { ns }\end{array}$ & $0,04 \mathrm{~ns}$ & $0,00058 \mathrm{~ns}$ & $0,02 \mathrm{~ns}$ & $0,00097 \mathrm{~ns}$ & $0,02 \mathrm{~ns}$ & $0,01 \mathrm{~ns}$ & $0,00015 \mathrm{~ns}$ & $0,01 \mathrm{~ns}$ & $0,0022 \mathrm{~ns}$ \\
\hline Error & 93 & 0,20 & 0,02 & 0,04 & 0,01 & 0,01 & 0,02 & 0,03 & 0,03 & 0,02 & 0,01 & 0,01 \\
\hline Total & 127 & & & & & & & & & & & \\
\hline C.V. $(\%)$ & & 9,87 & 18,22 & 22,63 & 8,54 & 13,95 & 18,02 & 16,01 & 11,57 & 7,44 & 6,03 & 8,05 \\
\hline
\end{tabular}

$n s=$ no significativo, ${ }^{*}=$ significativo, ${ }^{* *}=$ altamente significativo 
Concentración de esporas de Jorge tuvo la concentración más baja de micorrizas 831,81 esporas/100gss. Este resultado

El ADEVA realizada para esta variable sólo mostró diferencias estadísticas altamente significativas para localidades, destacándose El Chontal con un valor de 4169,75 esporas/100 gss, mientras que San podría ser una de las causas del mayor rendimiento exhibido en El Chontal. Las morfo-especies predominantes de hongos micorrícicos arbusculares en todas las localidades fueron Glomus y Acaulospora.

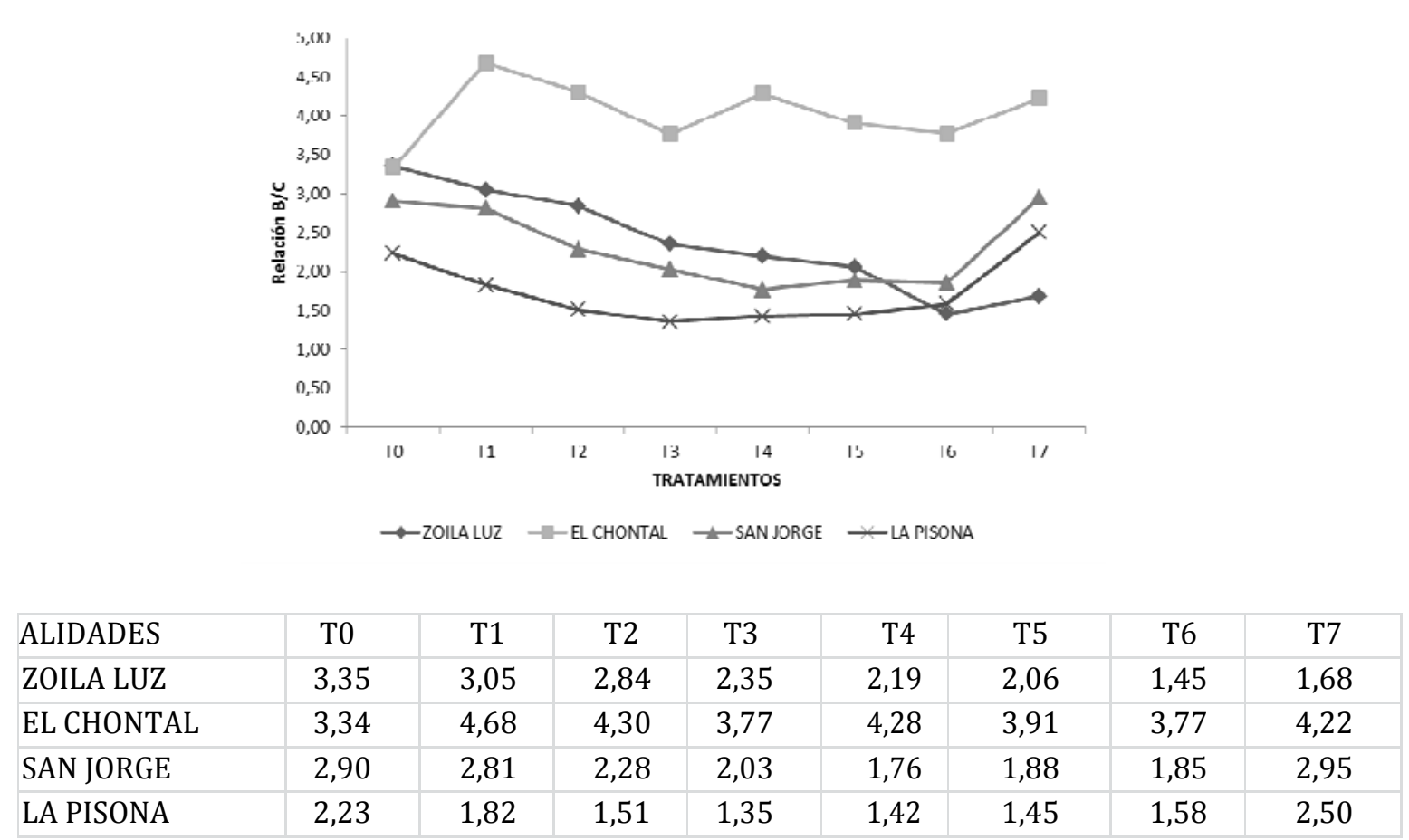

Figura 8. Relación Beneficio/Costo de los diferentes tratamientos donde se aplicaron las MPM en el cultivo de palmito.

\section{CONCLUSIONES}

En el análisis beneficio/costo se determinó que todos los tratamientos tienen un valor positivo y superior a 1 ; es decir, todos los tratamientos generarían un margen de utilidad. La localidad que reportó los mejores rendimientos y por tanto una mayor relación beneficio/costo fue El Chontal. En la mayoría de localidades hay una tendencia consistente, a medida que se iban implementando las MPM subían los costos variables disminuyendo la utilidad y por ende la relación B/C, excepto el tratamiento T7 que superó a todos los tratamientos, empero en la localidad de Zoila Luz el testigo (T0) tuvo la relación B/C más alta (Figura 8). Es factible que a mediano plazo al lograr un equilibrio en el desarrollo del cultivo y mejoras en el balance de nutrientes en el suelo se logre lo anotado por Tepper (2005), quien comenta que con el uso eficiente de las MPM se puede lograr una buena producción, ganancias y hasta ahorro que compensa el aumento de los costos de producción. 


\section{REFERENCIAS}

Bruuselma, T. C., F. Witt, S. García, T. Li, N. Rao, F. Chen y S. Ivanova. (2008). Un marco global para las mejores prácticas de manejo (MPM) de los fertilizantes. Informaciones agronómicas 38:1-4

Correndo, A. y García F. (2012). Concentración de nutrientes en planta como herramienta de diagnóstico: cultivos extensivos. Archivo agronómico IPNI 14: 1-8

Malavolta, E. (1994). Nutrición y fertilización del Maracuyá. International Plan Nutrition Institute. p. 32

Molina, E. (2000). Nutrición y fertilización del pejibaye para palmito. Disponible en:

http://www,ipni,net/publication/ialahp, nsf/0/624696A10165D977852579A300 79A77/\$FILE/Inf- Agro38.pdf

Mora, J. y Gainza, J. (1999). Palmito de pejibaye (Bactrisgasipaes Kunth) $\mathrm{Su}$ cultivo e industrialización. Disponible en:

http://books.google.com.ec/books/about /Palmito_de_pejibaye_Bactris_gasipaes_K un.html?id=gjxTOm G2zgYC\&redir_esc=y

Reyes, R., Pérez J., Peña, E., y Arcila, B. (2003). Deshije: Practica cultural para el cultivo de chontaduro (Bactris gasipaes K.) para palmito. Revista
Novedades Técnicas (Colombia). v.3. (3) p. 37-38

Silva, F. (2001). Fertilidad de suelos. Diagnóstico y control. Sociedad Colombiana de la Ciencia del Suelo. Segunda Edición. Bogotá. p. 59

Solano, R. (2012). Tesis de pregrado: "Efecto de la fertilización con NPK, sobre el desarrollo, estado nutritivo y rendimiento de plantas de palmito (Bactris gasipaes Kunth) inoculadas con micorrizas arbusculares nativas, en Santo Domingo

Tepper, P. (2005). Buenas Prácticas Agrícolas (BPA) y Manejo Integrado de Plagas (MIP). Disponible en: www.avocadosource.com/books/Ripa2 008/Ripa_Chapter_10.pdf

Villaprado, A. (2009). Evaluación de tres niveles de: nitrógeno, fósforo y potasio en el cultivo de palmito (Bactris gasipaes Kunt.) en producción, en el Cantón Puerto Quito. Escuela Politécnica del Ejército Santo Domingo, (http: //repositorio.espe.edu.ec/bitstream/210 00/4280/1/T-ESPE-IASA\%20II002283.pdf, (consultado 2 de septiembre de 2016) 